\title{
Augmenting Traditional ER Method to Support Spatiotemporal Database Applications
}

\author{
Sheng Lin, Peiquan Jin, Lei Zhao, Huaishuai Wang, and Lanlan Zhang \\ School of Computer Science and Technology, \\ University of Science and Technology of China, 230027, Hefei, China \\ jpq@ustc.edu.cn
}

\begin{abstract}
In this paper, we present a new conceptual modeling tool, called STXER (SpatioTemporal eXtended Entity-Relationship), to support spatiotemporal database applications. STXER provides most functions for database conceptual modeling, such as graphical user interface, SQL script generation, XML schema definition, and commercial-DBMS-oriented database creation. Compared with previous tools, STXER is ER-compatible and supports richer spatiotemporal semantics. Thus it can support both non-spatiotemporal and spatiotemporal applications. After an overview of the general features of STXER, we discuss the architecture of STXER. And finally, a case study of STXER's demonstration is presented.
\end{abstract}

\section{Introduction}

Previous works in spatiotemporal database were mainly focused on spatiotemporal data models, spatiotemporal indexes, and spatiotemporal query processing. Recently, a few spatiotemporal database prototype systems were proposed [1,2], in which they proposed to implement a spatiotemporal DBMS based on a commercial objectrelational DBMS, such as Oracle, DB2, or Informix. However, few works have been done in spatiotemporal conceptual modeling, which has been an obstacle to build spatiotemporal database applications on the basis of spatiotemporal DBMSs. As traditional conceptual modeling methods such as ER model can not support the modeling of spatiotemporal semantics, it is important to develop a spatiotemporal conceptual modeling tool to support spatiotemporal database applications.

The key challenge to develop a spatiotemporal conceptual modeling tool is how to support various spatiotemporal semantics. The commonly-used ER method fails to represent spatiotemporal entities and relationships. For example, the lifecycle of a geographical object, which refers to its creation, evolution, and elimination, is hard to be modeled according to the ER method. Recently, researchers have proposed some techniques to support spatiotemporal semantics in conceptual modeling tools. Since the ER model was widely used in the conceptual modeling of relational database applications, many people extended the ER model to support spatiotemporal applications [3-7]. For example, N. Tryfona et al [3] presented the STER method for spatiotemporal conceptual modeling. It used some special symbols and syntax to add spatiotemporal characteristics. This method, to our knowledge, is the only one that considered the topological changes into the framework. However, STER does not support modeling of 
objects' lifecycle. Besides, STER is very abstractive and no CASE tools have been implemented. The MADS tool [4] is much like the ER model, but it is not ERcompatible and does support rich spatiotemporal characteristics, e.g., the topological change is not supported. The DISTIL tool $[5,6]$ is ER-compatible but does support lifecycle and topological change.

In this paper, we present a conceptual modeling tool for spatiotemporal database applications, called STXER. The motivation of STXER is to design an effective tool for spatiotemporal applications so that they can create spatiotemporal database schemas in a traditional way used in relational database setting. A preliminary assumption of STEXR is that the fundamental spatiotemporal DBMS is built on a commercial object-relational DBMS such as Oracle and Informix. This assumption is reasonable and practical, as the abstract-type-based approach to implementing a practical spatiotemporal DBMS has been widely studied and promoted since it is proposed by R. H. Güting et al. in 2000 [7]. For example, spatiotemporal extensions on Oracle were proposed by different researchers in recent years $[1,2]$. Compared with previous spatiotemporal conceptual modeling methods, STXER has the following unique features:

(1) It is ER-compatible and offers a graphical user interface similar to traditional ER modeling tools, thus it is easy for users to develop spatiotemporal or nonspatiotemporal conceptual models.

(2) It supports richer spatiotemporal semantics than previous methods, so it can support diverse spatiotemporal database applications.

\section{Features of STXER}

STXER supports four types of spatiotemporal changes, which are described as follows:

(1) TYPE 1 (spatial processes): the spatial attributes of an object change with time, such as spread of fire, flowing of flood, moving of a car, or boundary changes of a land.

(2) TYPE 2 (thematic processes): the thematic attributes of an object change with time, such as changes of soil type and ownership change of a land.

(4) TYPE 3 (lifecycle): changes that result in creation of new objects or deletion of existing objects, such as split of a land or mergence of several lands.

(6) TYPE 4 (topological changes): changes of the topo-logical relationships of two related objects. For example, the topological relationship between a moving car and a road will change with time.

STXER extends traditional ER model in three aspects. First, it adds spatiotemporal types in the type system, so users can use those types to represent spatial or thematic processes (TYPE 1 and 2). Second, it adds a new attribute called $L C$ to represent the lifecycle of a spatiotemporal object (TYPE 3). Third, it introduces a new type of relationship into the ER model, called transformation, to represent the topological changes (TYPE 4).

STXER contains three components (as shown in Fig.1): 
(1) Graphical User Interface: This module provides a graphical user interface for users to define an STXER diagram. Fig.2 shows a snapshot of the user interface, which consists of a menu, a toolbox, a model tree view, and a diagram area. We also develop an effective algorithm to arrange the graphical elements in the diagram area.

The most difficult work in the graphical user interface is to adjust the connected lines between entities according to users' operations such as drag and drop of an entity, sizing an entity, or moving a line. We develop a partition-based algorithm in STXER to adjust the layout of lines when the graphical elements of the model are modified. According to this algorithm, the area related with two connected entities is divided into six sub-areas, namely a vertical sub-area, a horizontal sub-area, a southwestern sub-area (SW), a north-eastern sub-area (NE), a west-northern sub-area (NW), and a south-eastern sub-area (SE). The vertical and horizontal sub-areas always contain only straight lines, while the SW, NE, NW, and SE sub-areas always contain only polylines with one right angle.

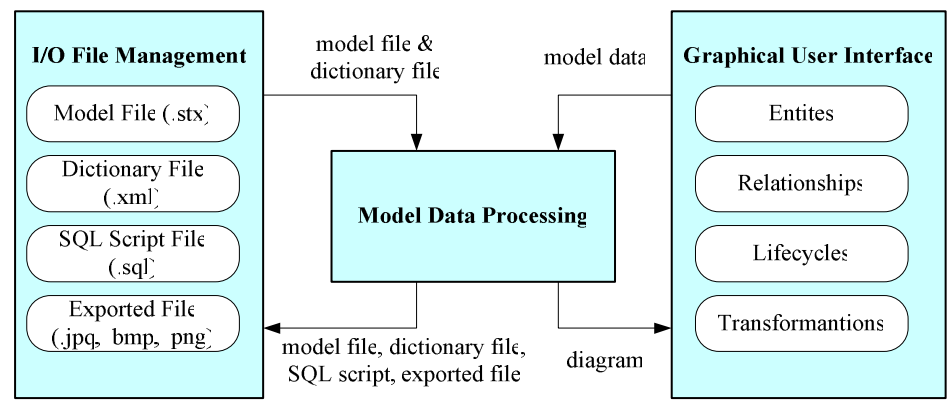

Fig. 1. Architecture of STXER

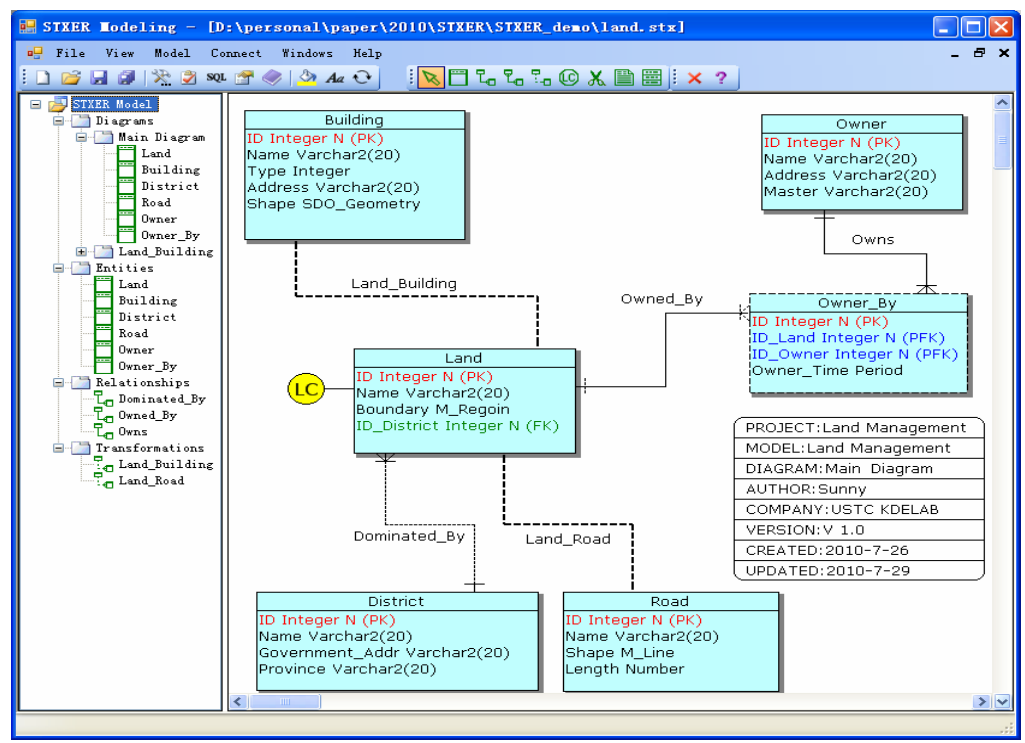

Fig. 2. The graphical user interface of STXER 
(2) Model Data Processing: In this module, we check the STXER model, and then the legal model can be further processed to generate SQL script or export metadata. During the checking process, we focus on three types of collisions, namely entity collision, relationship collision, and attribute collision.

(3) I/O File Management: In this module, we define the input and output format of the STXER model. The STXER model is finally saved as a .stx file, and the diagrams can be exported as .jpg, .bmp, or .png files. The metadata of an STXER model is maintained in a XML file. The XML file maintains information about the entities, relationships, transformations as well other elements of an STXER model. The XML file can be further used to generate a SQL script defining a physical database schema. Currently, STXER only allows users to generate the SQL script for Oracle $10 \mathrm{~g}$.

\section{Demonstration}

STXER is implemented in C\# language under Windows XP Professional and follow the object-oriented programming standard. Our demonstration will use STXER to create a spatiotemporal conceptual model for a land-use application and then build a spatiotemporal database in Oracle $10 \mathrm{~g}$. We will show how to quickly create an STXER diagram using our tool and represent different types of spatiotemporal semantics. In the demonstration, we will first present an overview of STXER and briefly discuss the modeling process of STXER. Then we will choose Oracle $10 \mathrm{~g}$ as the destination DBMS and connect to the Oracle database server. After that, we will use STXER to create spatiotemporal entities, relationships, lifecycles, and transformations to define an STXER model for a land-use application. We will also show how to check the STXER model and export XML schema and SQL scripts.

\section{Acknowledgements}

This work is supported by the National High Technology Research and Development Program ("863" Program) of China (No. 2009AA12Z204), the National Science Foundation of China (no. 60776801), the Open Projects Program of National Laboratory of Pattern Recognition (20090029), the Key Laboratory of Advanced Information Science and Network Technology of Beijing (xdxx1005), and the USTC Youth Innovation Foundation.

\section{References}

1. Jin, P., Sun, P.: OSTM: a Spatiotemporal Extension to Oracle. In: Proc. Of NCM 2008, pp. 575-580. IEEE CS Press, Los Alamitos (2008)

2. Pelekis, N., Theodoridis, Y.: An Oracle Data Cartridge for Moving Objects, Technical Report, UNIPI-ISL-TR-2007-04, Dept. of Informatics, Univ. of Piraeus (2007)

3. Tryfona, N., Price, R., Jensen, C.: Conceptual Models for Spatio-temporal Applications. In: Sellis, T.K., Koubarakis, M., Frank, A., Grumbach, S., Güting, R.H., Jensen, C., Lorentzos, N.A., Manolopoulos, Y., Nardelli, E., Pernici, B., Theodoulidis, B., Tryfona, N., Schek, H.J., Scholl, M.O. (eds.) Spatio-Temporal Databases. LNCS, vol. 2520, pp. 79-116. Springer, Heidelberg (2003) 
4. Parent, C., Spaccapietra, S., et al.: Modeling Spatial Data in the MADS Conceptual Model. In: Proc. of SDH (1998)

5. Ram, S., Snodgrass, R., et al.: DISTIL: A Design Support Environment for Conceptual Modeling of Spatio-temporal Requirements. In: Kunii, H.S., Jajodia, S., Sølvberg, A. (eds.) ER 2001. LNCS, vol. 2224, pp. 70-83. Springer, Heidelberg (2001)

6. Khatri, V., Ram, S., Snodgrass, R.: On Augmenting Database Design-support Environments to Capture the Geo-spatiotemporal Data Semantics. Information System 31(2), 98-133 (2006)

7. Güting, R.H., Böhlen, M.H., Erwig, M., et al.: A Foundation for Representing and Querying Moving Objects. ACM Transactions on Database Systems 25(1), 1-42 (2000) 